\title{
ON DEFICIENCY INDEX FOR SOME SECOND ORDER VECTOR DIFFERENTIAL OPERATORS
}

\author{
I.N. BRAEUTIGAM, K.A. MIRZOEV, T.A. SAFONOVA
}

\begin{abstract}
In this paper we consider the operators generated by the second order matrix linear symmetric quasi-differential expression

$$
l[y]=-\left(P\left(y^{\prime}-R y\right)\right)^{\prime}-R^{*} P\left(y^{\prime}-R y\right)+Q y
$$

on the set $[1,+\infty)$, where $P^{-1}(x), Q(x)$ are Hermitian matrix functions and $R(x)$ is a complex matrix function of order $n$ with entries $p_{i j}(x), q_{i j}(x), r_{i j}(x) \in L_{l o c}^{1}[1,+\infty)(i, j=$ $1,2, \ldots, n)$. We describe the minimal closed symmetric operator $L_{0}$ generated by this expression in the Hilbert space $L_{n}^{2}[1,+\infty)$. For this operator we prove an analogue of the Orlov's theorem on the deficiency index of linear scalar differential operators.
\end{abstract}

Keywords: Quasi-derivative, quasi-differential expression, minimal closed symmetric differential operator, deficiency numbers, asymptotic of the fundamental system of solutions.

Mathematics Subject Classification: 34A30, 34L05, 47E05

\section{INTRODUCTION}

In [1] S.A. Orlov found a class of linear symmetric differential operators with real analytic coefficients, whose deficiency numbers are determined as the number of roots in the left halfplane of some explicitly written polynomial. Namely, the following theorem was given.

Theorem 1. Let functions $p_{0}, p_{1}, \ldots, p_{m}$ be defined on the set $[1,+\infty)$ and satisfy the conditions:

(I) $p_{0}, p_{1}, \ldots, p_{m}$ are measurable, real-valued and for each $b \in(1,+\infty)$

$$
\int_{1}^{b}\left|p_{m}\right|^{-1}<+\infty, \int_{1}^{b}\left|p_{k}\right|<+\infty \quad(k=0,1, \ldots, m-1),
$$

(II) $p_{0}(z), p_{1}(z), \ldots, p_{m}(z)$ are analytic functions as $|z| \geqslant x_{0} \geqslant 1$ and

$$
p_{k}(z)=z^{2 k+\nu}\left[a_{k}+\sum_{j=1}^{+\infty} a_{j}^{(k)} z^{-j}\right] \quad\left(k=0,1, \ldots, m ;|z| \geqslant x_{0} \geqslant 1\right),
$$

where $a_{m} \neq 0$, and $\nu \geqslant 0$ is an integer number. Then the maximal number of linear independent solutions to the equation

$$
l_{2 m}[y](x):=p_{0}(x) y+\frac{d}{d x}\left\{p_{1}(x) y^{\prime}+\frac{d}{d x}\left[p_{2}(x) y^{\prime \prime}+\ldots\right.\right.
$$

I.N. Braeutigam, K.A. Mirzoev, T.A. Safonova, On Deficiency index for SEcond order vector DIFFERENTIAL OPERATORS.

(c) Braeutigam I.N., Mirzoev K.A., Safonova T.A. 2017.

The first author is supported by the grant of the Ministery of Educations and Science of Russia and German Academic Exchange Service (DAAD) under the program "Mikhail Lomonosov' (no. 1.728.2016/DAAD). The second author is supported by the grant of RSF (no. 14-11-00754). The third author is supported by Ministery of Educations and Science of Russia (the grant of the President of Russia no. MK-3941.2015.1).

Submitted May 24, 2016. 


$$
\left.\left.\ldots+\frac{d}{d x}\left(p_{m-1}(x) y^{(m-1)}+\frac{d}{d x}\left(p_{m}(x) y^{(m)}\right)\right) \ldots\right]\right\}=\lambda y,
$$

belonging to $L^{2}[1,+\infty)$ is equal

1) as $\nu>0$, to the number of the roots of the polynomial

$$
F_{2 m}(z, \nu)=\sum_{k=1}^{m} a_{k} \prod_{j=0}^{k-1}\left[\left(z+\frac{\nu}{2}\right)^{2}-\left(\frac{\nu+1}{2}+j\right)^{2}\right]+a_{0}
$$

lying in the domain $\operatorname{Re} z<0$ and this number is independent of $\lambda$. At that, the spectrum of each self-adjoint extension of the corresponding operator is discrete.

2) as $\nu=0$, to the number of the roots of the polynomial $F_{2 m}(z, 0)-\lambda$ lying in the domain $\operatorname{Re} z<0$ and for non-real $\lambda$, it is equal to $m$.

Assume that the functions $p_{0}(x), p_{1}(x), \ldots, p_{m}(x)$ are represented as

$$
p_{k}(x)=x^{2 k+\nu}\left(a_{k}+r_{k}(x)\right) \quad(k=0,1, \ldots, m-1), \quad p_{m}(x)=\frac{x^{2 m+\nu}}{\frac{1}{a_{m}}+r_{m}(x)},
$$

where $\nu$ is a non-negative (not necessarily integer) number; $a_{0}, a_{1}, \ldots, a_{m}$ are real number, $a_{m} \neq 0$; and $r_{0}, r_{1}, \ldots, r_{m}$ are real functions on $[1,+\infty)$ such that

i) for some $x_{0}(\geqslant 1)$

$$
\int_{x_{0}}^{+\infty}\left|r_{k}(x)\right| \frac{d x}{x}<+\infty \quad(k=0,1, \ldots, m)
$$

ii) all the roots of the polynomial $F_{2 m}(z, \nu)$ as $\nu>0$ and of the polynomial $F_{2 m}(z, 0)-\lambda$ are different.

In work [2], F.A. Neimark established in particular that Statements 1) and 2) of Theorem 1 are still true if we keep Condition (I) and replace Condition (II) by Conditions i) and ii). Later in the joint work [3] by R.B. Paris and A.D. Wood, Theorem 1 was discovered once again for the particular case $p_{k}(x)=a_{k} x^{2 k+\nu}(k=0,1, \ldots, m)$, where $\nu$ is a non-negative integer number and the polynomial $F_{2 m}(z, \nu)$ was studied in details. In particular, by this method they established that there exist positive numbers $K$ and $\nu$ such that the deficiency index of the minimal closed symmetric operator generated by the expression

$$
l_{6}[y]=-\left(x^{6+\nu} y^{(3)}\right)^{(3)}+K x^{\nu} y
$$

is equal to $(5,5)$ and they specified in this way the results by R.M. Kauffman [4] stating that the deficiency index of this operator is not $(3,3)$.

Later the results of work [3] were involved in book [5], in which there were also considered some differential operators generated by particular expressions of odd order and the associated polynomials were studied. It seems that works [1] and [2] remained unknown to the authors of work [3] and book [5].

In [6], K.A. Mirzoev studied the deficiency index and the character of the spectrum of the minimal closed symmetric operator generated by the quasi-differential expression $l_{n}$ of arbitrary (even or odd) order $n$ with complex-valued coefficients on the set $[1 ;+\infty)$. The obtained results are similar to the statements in the Orlov theorem. At that, the conditions for the coefficients of the expression $l_{n}$ are of the same nature as Condition (I) in Theorem 1, while Condition i) is changed so that Condition ii) is no longer needed.

In joint work [7], I.N. Dolgikh (I.N. Braeutigam) and K.A. Mirzoev considered a similar problem both on the half-line and the interval $(0,1]$, that is, they widened essentially the class of the operators, for which Orlov theorem is still true. 
Our aim is to construct the spectral theory of differential operators generated by formally self-adjoint expressions

$$
l[y]=-\left(P\left(y^{\prime}-R y\right)\right)^{\prime}-R^{*} P\left(y^{\prime}-R y\right)+Q y
$$

in the space $L_{n}^{2}[1,+\infty)$, where $P, Q, R$ are complex-valued matrix functions of order $n(n \in \mathbb{N})$ defined on the ray $[1,+\infty)$ such that $P$ is non-degenerate, $P$ and $Q$ are Hermitian matrices and the entries of the matrix functions $P^{-1}, Q$ and $R$ are measurable on $[1,+\infty)$ and summable on each its closed finite subinterval. Our aim is also to establish an analogue of Orlov theorem and to construct examples realizing the limit-point case and limit-circle case for such operators

$$
l[y]=-\left(\mathcal{P}_{0} y^{\prime}\right)^{\prime}+i\left(\left(\mathcal{Q}_{0} y\right)^{\prime}+\mathcal{Q}_{0} y^{\prime}\right)+\mathcal{P}_{1}^{\prime} y .
$$

Here the derivatives are treated in the sense of distributions theory, $\mathcal{P}_{0}, \mathcal{Q}_{0}$ and $\mathcal{P}_{1}$ are Hermitian matrix functions of order $n$ with Lebesgue measurable entries such that $\mathcal{P}_{0}^{-1}$ exists and $\left\|\mathcal{P}_{0}\right\|,\left\|\mathcal{P}_{0}^{-1}\right\|,\left\|\mathcal{P}_{0}^{-1}\right\|\left\|\mathcal{P}_{1}\right\|^{2},\left\|\mathcal{P}_{0}^{-1}\right\|\left\|\mathcal{Q}_{0}\right\|^{2} \in L_{l o c}^{1}[1,+\infty)$

The rigorous definition of the expression $l$ as well as of the minimal closed symmetric operator generated by this expression are given in Section 2.

A part of the results of this work was published without proofs in [8].

\section{Quasi-Derivatives and quASI-Differential operators. DeficienCy indices}

2.1. Let $I:=[1,+\infty)$ and $P(x), Q(x)$ and $R(x)$ be square matrix functions of order $n(n \in \mathbb{N})$ defined on the set $I$ and $P(x)$ is non-degenerate, $P(x)$ and $Q(x)$ are Hermitian matrices as $x \in I$ such that the following condition holds.

Condition A. The complex-valued functions $p_{i j}, q_{i j}$ and $r_{i j}(i, j=1,2, \ldots, n)$, the entries of the matrices $P^{-1}, Q$ and $R$, respectively, are defined and measurable on the set $I$ and summable on each its closed subinterval, that $i s, p_{i j}, q_{i j}, r_{i j} \in L_{l o c}^{1}(I)$.

By the symbol $A C_{l o c}(I)$ we denote the set of all vector functions $y(x)=\left(y_{1}(x), y_{2}(x), \ldots, y_{n}(x)\right)^{t}$, where $t$ stands for the transposition, with locally absolutely continuous entries on $I$ and we introduce the first quasi-derivative of a given vector function $y \in A C_{l o c}(I)$ as

$$
y^{[1]}:=P\left(y^{\prime}-R y\right) \text {. }
$$

Assuming that the vector function $y^{[1]}$ is defined and $y^{[1]} \in A C_{l o c}(I)$, we introduce the second quasi-derivative of the vector function $y$ as

$$
y^{[2]}:=\left(y^{[1]}\right)^{\prime}+R^{*} y^{[1]}-Q y,
$$

where $^{*}$ stands for the adjoint symbol, and we introduce the quasi-differential expression

$$
l[y](x):=-y^{[2]}(x), \quad x \in I .
$$

Thus,

$$
l[y]=-\left(P\left(y^{\prime}-R y\right)\right)^{\prime}-R^{*} P\left(y^{\prime}-R y\right)+Q y,
$$

and the set of vector functions $\mathcal{D}:=\left\{y(x) \mid y(x), y^{[1]}(x) \in A C_{l o c}(I)\right\}$ is obviously the domain of this expression. It follows from Condition $(A)$ that for each vector function $y(x) \in \mathcal{D}$ the expression $l[y](x)$ exists almost everywhere on $I$ and the entries of $l[y]$ are locally integrable. Moreover, for each two vector functions $f, g \in \mathcal{D}$ we have the following lemma, which is a vector analogue of the Green identity.

Lemma 1. Let $P, Q$ and $R$ be square matrix functions of order $n$ satisfying all aforementioned conditions on $I$. Then for each two vector functions $u, v \in \mathcal{D}$ and each two numbers $\alpha$ 
and $\beta$ such that $0 \leqslant \alpha \leqslant \beta<\infty$ the formula

$$
\int_{\alpha}^{\beta}\{(l[u](x), v(x))-(u(x), l[v](x))\} d x=[u(x), v(x)](\beta)-[u(x), v(x)](\alpha)
$$

holds, where $(g, h)=\sum_{s=1}^{n} g_{s} \overline{h_{s}}$ is the scalar product of the vectors $g$ and $h$, while the form $[u, v]$ is defined by the identity

$$
[u, v](x):=\left(u^{[1]}(x), v(x)\right)-\left(u(x), v^{[1]}(x)\right) .
$$

In [9], Lemma 1 was proved for a particular case of the expression $l$, when $P(x)=I_{n}\left(I_{n}\right.$ is the unit matrix of order $n), R(x)=\sigma(x), Q(x)=-\sigma^{2}(x)$, where $\sigma(x)$ is a given symmetric matrix function of order $n$ with real entries such that the entries of the matrix $\sigma^{2}(x)$ are locally integrable in $I$. The proof given in this work can be extended to the expression $l$ of form (1) with no major changes.

Let $L_{n}^{2}(I)$ be the space of the equivalence classes of all complex-valued measurable vector functions $y$ such that the sum of the squares of their entries are Lebesgue measurable in $I$. In the literature devoted to the spectral theory of ordinary differential operators, the procedure of determining the minimal operator $L_{0}$ generated by the expression $l[y]$ in the Hilbert space $L_{n}^{2}(I)$ is well-known. Namely, denoting by $D_{0}^{\prime}$ the set of all complex-valued compactly supported on $I$ vector functions in $\mathcal{D}$ such that $l[y] \in L_{n}^{2}(I)$, arguing as in the scalar case (see, for instance, [10]) and using Green formula (2), we establish that the set $D_{0}^{\prime}$ is everywhere dense in $L_{n}^{2}(I)$ and by the formula $L_{0}^{\prime} y=l[y]$, the expression $l$ defines a symmetric (unclosed) operator in $L_{n}^{2}(I)$ with the domain $D_{0}^{\prime}$. By the symbols $L_{0}$ and $D_{0}$ we denote the closure of this operator and its domains, respectively. Thanks to this, similar to the concept of scalar symmetric quasidifferential expression, in what follows the expression $l$ is referred to as (formally self-adjoint) the quasi-differential expression generated by means of the matrices $P, Q$ and $R$.

Let $\lambda$ be a complex number and $\operatorname{Im} \lambda \neq 0$. By $R_{\lambda}$ and $R_{\bar{\lambda}}$ we denote the domains of the operators $L_{0}-\lambda \mathcal{I}$ and $L_{0}-\bar{\lambda} \mathcal{I}$, respectively, while $\mathcal{N}_{\lambda}$ and $\mathcal{N}_{\bar{\lambda}}$ stand for the orthogonal complements of the spaces $R_{\bar{\lambda}}$ and $R_{\lambda}$ in $L_{n}^{2}(I)$. The spaces $\mathcal{N}_{\lambda}$ and $\mathcal{N}_{\bar{\lambda}}$ are called defect spaces, the numbers $n_{+}$and $n_{-}$equal to their dimensions, $n_{+}=\operatorname{dim} \mathcal{N}_{\lambda}, n_{-}=\operatorname{dim} \mathcal{N}_{\bar{\lambda}}$, are called deficiency numbers of the operator $L_{0}$ in the upper and lower half-planes, respectively, while the pair $\left(n_{+}, n_{-}\right)$is called the deficiency index of the operator $L_{0}$.

Arguing similar to [11] and [12, one can establish that the numbers $n_{+}$and $n_{-}$coincide with the maximal number of linearly independent solutions to the equation

$$
l[y]=\lambda y
$$

belonging to the space $L_{n}^{2}(I)$ as the parameter $\lambda$ ranges in the upper $(\operatorname{Im} \lambda>0)$ or in the lower $(\operatorname{Im} \lambda<0)$ half-plane, respectively. These numbers satisfy the double inequality $n \leqslant n_{+}$, $n_{-} \leqslant 2 n$, and $n_{+}=2 n$ if and only if $n_{-}=2 n$. Moreover, the case $n_{+}=n_{-}=2 n$ is realized if and only if all solutions to equation (3) belong to the space $L_{n}^{2}(I)$ for all $\lambda \in \mathbb{C}$. Using the analogy with the spectral theory of scalar Sturm-Liouville operators on the half-line, one sometimes says that the expression $l[y]$ (for the operator $L_{0}$ ) is in the limit-point case occurs if $n_{+}=n_{-}=n$, while as $n_{+}=n_{-}=2 n$, one says that the expression $l[y]$ (for the operator $L_{0}$ ) in the limit-circle case (see, for instance, [11]).

Equation (3) is equivalent to the system of first order ordinary differential equations

$$
\mathbf{y}^{\prime}=(F-\Lambda) \mathbf{y}
$$


where $\mathbf{y}=\left(\begin{array}{c}y \\ y^{[1]}\end{array}\right)$, the matrices $F$ and $\Lambda$ are of order $2 n$ and have the form

$$
F=\left(\begin{array}{cc}
R & P^{-1} \\
Q & -R^{*}
\end{array}\right), \quad \Lambda=\left(\begin{array}{cc}
O & O \\
\lambda I_{n} & O
\end{array}\right)
$$

while, as usually, $O$ and $I_{n}$ are the zero and unit matrix of order $n$, respectively.

Equations (3) and (4) are equivalent in the sense that if $y(x)$ is a vector solution of system (3), then the vector column $\mathbf{y}$ solves equation (4) and vice versa, if $2 n$-dimensional vector column $\mathbf{y}$ is a solution of system (4), then the vector $y$ formed by first $n$ entries of the vector $\mathbf{y}$ solves equation (3).

Remark 1. The conditions on the entries of the matrices $P, Q$ and $R$ ensure the unique solvability of the Cauchy problem for system (4) posed at arbitrary point of the set I and are the most general conditions ensuring such solvability, see [13, Ch. 1, Th. 1.2.3]. The equivalence of systems (3) and (4) imply the unique solvability for system (3).

Employing the terminology from the theory of operators generated by linear differential expressions with non-smooth coefficients, one sometimes says that the quasi-derivatives $y^{[0]}(:=$ $y), y^{[1]}, y^{[2]}$ and the quasi-differential expression $l[y]$ are generated by the matrix $F$.

2.2. Let $\mathcal{P}_{0}, \mathcal{Q}_{0}$ and $\mathcal{P}_{1}$ are Hermitian matrix functions of order $n$ with measurable entries such that $\mathcal{P}_{0}^{-1}$ exists and $\left\|\mathcal{P}_{0}^{-1}\right\|,\left\|\mathcal{P}_{0}^{-1}\right\|\left\|\mathcal{P}_{1}\right\|^{2},\left\|\mathcal{P}_{0}^{-1}\right\|\left\|\mathcal{Q}_{0}\right\|^{2}$ are locally Lebesgue integrable. Let $\varphi:=\mathcal{P}_{1}+i \mathcal{Q}_{0}$ and $\varphi^{*}:=\mathcal{P}_{1}-i \mathcal{Q}_{0}$. We consider the block matrix

$$
\mathcal{F}=\left(\begin{array}{cc}
\mathcal{P}_{0}^{-1} \varphi & \mathcal{P}_{0}^{-1} \\
-\varphi^{*} \mathcal{P}_{0}^{-1} \varphi & -\varphi^{*} \mathcal{P}_{0}^{-1}
\end{array}\right)
$$

Employing the properties of the matrix norms and the hermiticity of the matrix function $\mathcal{P}_{0}$, $\mathcal{Q}_{0}$ and $\mathcal{P}_{1}$, it is easy to confirm that all the entries of the matrix $\mathcal{F}$ belong to the space $L_{\text {loc }}^{1}(I)$.

By means of the matrix $\mathcal{F}$ we introduce the quasi-derivatives $y^{[0]}, y^{[1]}, y^{[2]}$ letting as before

$$
y^{[0]}=y, \quad y^{[1]}=\mathcal{P}_{0} y^{\prime}-\varphi y, \quad y^{[2]}=\left(y^{[1]}\right)^{\prime}+\varphi^{*} \mathcal{P}_{0}^{-1} y^{[1]}+\varphi^{*} \mathcal{P}_{0}^{-1} \varphi y .
$$

Applying Remark 1, we conclude that the theorem on the unique solvability of the Cauchy problem posed at an arbitrary point in $I$ holds true for the equation

$$
-y^{[2]}=\lambda y \text {. }
$$

If we assume that the entries of the matrix $\mathcal{P}_{0}$ also belong to $L_{l o c}^{1}(I)\left(\left\|\mathcal{P}_{0}\right\| \in L_{l o c}^{1}(I)\right)$, then it is easy to see that the entries of the matrix $\varphi$ are locally integrable in $I$. Under these assumptions, one can prove that if we treat ' as the differentiation in the sense of the distribution theory, then in the expression $y^{[2]}$ we can open the brackets and we obtain the formula

$$
y^{[2]}=\left(\mathcal{P}_{0} y^{\prime}\right)^{\prime}-i\left(\left(\mathcal{Q}_{0} y\right)^{\prime}+\mathcal{Q}_{0} y^{\prime}\right)-\mathcal{P}_{1}^{\prime} y
$$

Thus, in terms of the distributions, the expression $l[y]$ (see (1D) is written as

$$
l[y]=-\left(\mathcal{P}_{0} y^{\prime}\right)^{\prime}+i\left(\left(\mathcal{Q}_{0} y\right)^{\prime}+\mathcal{Q}_{0} y^{\prime}\right)+\mathcal{P}_{1}^{\prime} y,
$$

and the operator $L_{0}$ introduced above can be treated as the operator generated by this expression in the Hilbert space $L_{n}^{2}(I)$. Such interpretation of the operator with the distribution coefficients allows us to include it into the class of the operators generated by quasi-differential expressions with locally summable coefficients in the space $L_{n}^{2}(I)$ and to construct the spectral theory of this operator.

We observe that a correct definition of the Sturm-Liouville operator with a first order scalar distribution potential, that is, of the operator generated by the expression of the form

$$
l[y]=-y^{\prime \prime}+\sigma^{\prime}(x) y
$$


in the space $L^{2}(a, b)$, where $\sigma$ is a complex-valued function such that $\sigma^{2} \in L_{l o c}^{1}(a, b)$, seems to be given first in some works by A.M. Savchuk and A.A. Shkalikov (see [14], [15]), while for the vector analogue of this expression, when $\sigma(x)$ is a square symmetric matrix function of order $n$ with real entries such that the entries of $\sigma^{2}$ are locally integrable in the half-line, such definition was given in [9].

In particular, if $\mathcal{Q}_{0}(x)=O$, then vector quasi-differential expression (5) casts into the form

$$
l[y]=-\left(\mathcal{P}_{0} y^{\prime}\right)^{\prime}+\mathcal{P}_{1}^{\prime} y .
$$

\section{Analogue of Orlov theorem}

3.1. In what follows we shall need the following lemma, see [16], [17, Ch. III, Problem 35].

Lemma 2. Consider the system of ordinary differential equations

$$
U^{\prime}=(A+G(t)) U
$$

where $A$ is a constant matrix, whose canonical form has Jordan blocks $J_{k}, k \geqslant 1$, and the maximal number of the rows for all the blocks $J_{k}$ is equal to $r+1$. Assume that

$$
\int_{1}^{\infty} t^{r}\|G(t)\| d t<\infty
$$

Let $z_{j}$ be a characteristic root of the matrix $A$ and let the equation $y^{\prime}=$ Ay has a solution of the form

$$
e^{z_{j} t} t^{k} c+O\left(e^{z_{j} t} t^{k-1}\right)
$$

where $c$ is a constant vector. Then equation (6) has the solution $\phi$ such that

$$
\phi(t)=e^{z_{j} t} t^{k}(c+o(1)) \quad \text { as } \quad t \rightarrow+\infty .
$$

3.2. In what follows we suppose that the matrix functions $P^{-1}, Q$ and $R$ are the coefficients of the expression $l[y]$, see (1), satisfy the following condition:

Condition B. For all $x \geqslant 1$ and some real $\nu \geqslant 0$

$$
P^{-1}(x)=x^{-\nu-2}\left(P_{0}+P_{1}(x)\right), \quad Q(x)=x^{\nu}\left(Q_{0}+Q_{1}(x)\right), \quad R(x)=x^{-1}\left(R_{0}+R_{1}(x)\right),
$$

where $P_{0}, Q_{0}, R_{0}$ and $P_{1}(x), Q_{1}(x), R_{1}(x)$ are Hermitian constant matrices and matrix functions of order $n$, respectively. Let $\operatorname{det} P_{0} \neq 0$ and moreover,

$$
\int_{1}^{+\infty} \frac{\ln ^{r} x}{x}\left(\left\|P_{1}(x)\right\|+\left\|Q_{1}(x)\right\|+\left\|R_{1}(x)\right\|\right) d x<+\infty,
$$

where $r+1$ is the maximal number of the rows for all Jordan blocks $J_{k}, k \geqslant 1$, in the canonical form of the matrix

$$
A_{\nu}:=\left(\begin{array}{cc}
R_{0}+\frac{1}{2} I_{n} & P_{0} \\
Q_{0}-\lambda \chi(\nu) I_{n} & -R_{0}^{*}-\left(\nu+\frac{1}{2}\right) I_{n}
\end{array}\right),
$$

where $\chi(\nu)=0$ as $\nu>0$ and $\chi(0)=1$.

The following theorem holds true.

Theorem 2. Let the elements $P^{-1}, Q$ and $R$ of the matrix $F$ satisfy Condition (B) and let $l$ be the quasi-differential expression generated by this matrix (see (1)). Then the maximal number of linearly independent solutions of equation (3) belonging to the space $L_{n}^{2}(I)$ is equal 1) as $\nu>0$, to the number of the roots of the polynomial $\mathcal{F}(z, \nu):=\operatorname{det}\left(A_{\nu}-z I_{2 n}\right)$ (counting the multiplicities) lying in the domain $\operatorname{Re} z<0$ and is independent of $\lambda$;

2) as $\nu=0$, to the number of the roots of the polynomial $\mathcal{F}(z, 0):=\operatorname{det}\left(A_{0}-z I_{2 n}\right)$ (counting the multiplicities) lying in the domain $\operatorname{Re} z<0$ and for non-real $\lambda$ it is equal to $n$. 
Moreover, in the case $\nu>0$, the spectrum of each self-adjoint extension of the operator $L_{0}$ is discrete.

Proof. It follows from Condition (B) that the entries of the matrix $F$ satisfy Condition (A) of Subsection 2.1. This is why due to formula (1), the quasi-differential expression $l[y]$ generated by this matrix is well-defined as well as the minimal closed symmetric operator $L_{0}$, and Statements 1 and 2 of Theorem 2 describe the deficiency numbers of this operator.

Let $\nu>0$. We denote by $D$ the block-diagonal matrix

$$
D=\left(\begin{array}{cc}
x^{-1 / 2} I_{n} & O \\
O & x^{\nu+1 / 2} I_{n}
\end{array}\right) .
$$

In system (4) we make the change $\mathbf{y}=D Y$, where $Y$ is a new unknown $2 n$-dimensional vector function. As the result, system (4) becomes

$$
Y^{\prime}=\left(D^{-1} F D-D^{-1} \Lambda D-D^{-1} D^{\prime}\right) Y .
$$

Simple calculations show that the matrix functions $D^{-1} F D, D^{-1} \Lambda D$ and $D^{-1} D^{\prime}$ in the block representation have the form

$$
\begin{aligned}
& D^{-1} D^{\prime}=x^{-1}\left(\begin{array}{cc}
-1 / 2 I_{n} & O \\
O & (\nu+1 / 2) I_{n}
\end{array}\right), \quad D^{-1} \Lambda D=x^{-1}\left(\begin{array}{cc}
O & O \\
\lambda x^{-\nu} I_{n} & O
\end{array}\right), \\
& D^{-1} F D=x^{-1}\left(\begin{array}{cc}
x R & x^{\nu+2} P^{-1} \\
x^{-\nu} Q & -x R^{*}
\end{array}\right) .
\end{aligned}
$$

Thus, the unknown function $Y$ satisfies the system of differential equations

$$
x Y^{\prime}=\left(A_{\nu}+B(x)\right) Y,
$$

where $A_{\nu}$ is the scalar matrix defined above and $B(x)$ is the matrix function

$$
B(x)=\left(\begin{array}{cc}
R_{1}(x) & P_{1}(x) \\
Q_{1}(x)-\frac{\lambda}{x^{\nu}} I_{n} & -R_{1}^{*}(x)
\end{array}\right) .
$$

Letting $x=e^{t}$, we observe that system (8) becomes (6), where $U(t)=Y\left(e^{t}\right), A=A_{\nu}$ and $G(t)=B\left(e^{t}\right)$.

We consider the system of differential equations with constant coefficients

$$
U^{\prime}=A_{\nu} U(t) .
$$

The fundamental matrix of the solutions of this system is $\Phi=e^{A_{\nu} t}$.

Let $z$ be a characteristic root of the matrix $A_{\nu}$ of algebraic multiplicity $r_{0}$ and geometric multiplicity $l$. We denote by $k_{i}(i=1,2 \ldots l)$ the dimension of Jordan blocks corresponding to the number $z$. We note that $2 n$-dimensional vector columns of the fundamental matrix $\Phi$ corresponding to $i$ th Jordan block are

$$
e^{z t} c_{k_{i}}, \quad t^{j} e^{z t} c_{k_{i}}+O\left(t^{j-1} e^{z t}\right), \quad j=1, \ldots, k_{i}-1,
$$

where $c_{k_{i}}$ is the eigenvector of the matrix $A$ corresponding to this Jordan block (for more details see [17, Ch. III, Sect. 4]).

Let $\left(\begin{array}{l}X_{1} \\ X_{2}\end{array}\right)$, where $X_{i}(i=1,2)$ are $n$-dimensional vector columns, be the eigenvector associated with the eigenvalue $z$ of the matrix $A_{\nu}$, that is,

$$
\left(A_{\nu}-z I_{2 n}\right)\left(\begin{array}{l}
X_{1} \\
X_{2}
\end{array}\right)=O
$$


Hence, the vectors $X_{1}$ and $X_{2}$ satisfy the system of equations

$$
\left\{\begin{array}{l}
\left(R_{0}+\left(\frac{1}{2}-z\right) I_{n}\right) X_{1}+P_{0} X_{2}=O \\
\left(-R_{0}^{*}-\left(\nu+\frac{1}{2}+z\right) I_{n}\right) X_{1}+Q_{0} X_{2}=O .
\end{array}\right.
$$

Taking into consideration that $\operatorname{det} P_{0} \neq 0$ and excluding the unknown vector $X_{2}$ from this system, we obtain that the vector $X_{1}$ satisfies the equation

$$
\left(-R_{0}^{*}-\left(\nu+\frac{1}{2}+z\right) I_{n}-Q_{0} P_{0}^{-1}\left(R_{0}+\left(\frac{1}{2}-z\right) I_{n}\right) X_{1}=O .\right.
$$

The vector $X_{1}$ is obviously non-zero and moreover, the geometric multiplicity of the root $z$ is equal to $l$. Hence, the rank of the matrix coefficient in system (10) is equal to $n-l(l \in$ $\{1,2 \ldots, n-1\})$. Thus, this system has $l$ linearly independent solutions, that is, $l$ eigenvectors of the form $\left(\begin{array}{l}X_{1} \\ X_{2}\end{array}\right)$ are associated with the characteristic root $z$ and first $n$ coordinates of these vectors do not vanish simultaneously.

Taking into consideration Condition (B), we observe that system (8) is reduced to system (6) and at that, (7) holds true, that is, all the conditions of Lemma 2 are satisfied. Applying this lemma to each function in list (9), we obtain that system (6) has a fundamental matrix of solutions formed by vector columns, which can be represented for $t \rightarrow \infty$ as

$$
t^{k} e^{z t}\left(c_{k_{i}}+o(1)\right) \quad\left(k=0,1, \ldots k_{i}-1\right) .
$$

Making the inverse change $x=e^{t}$ and taking into consideration that $\mathbf{y}=D Y$, we obtain that the vector columns of the fundamental matrix of equation (3) associated with the characteristic root $z$ of the matrix $A$ are of the form

$$
x^{z-\frac{1}{2}} \ln ^{k} x\left(\widetilde{c}_{k_{i}}+o(1)\right),
$$

where $\widetilde{c}_{k_{i}}$ are non-zero vectors consisting of first $n$ entries of the vectors $c_{k_{i}}$.

The functions, which can be represented as (11), belong to the space $L_{n}^{2}(I)$ if and only if

$$
\int_{1}^{+\infty}\left|x^{2 z-1}\right|(\ln x)^{2 k} d x<\infty,
$$

and this is true if and only if $\operatorname{Re} z<0$. Moreover, as $\nu>0$, the polynomial $\mathcal{F}(z, \nu)$ is independent of $\lambda$. Thus, the deficiency numbers of the operator $L_{0}$ coincide and are equal to the number of the roots of the equation $\mathcal{F}(z, \nu)=0$ satisfying the condition $\operatorname{Re} z<0$.

Then one can show that the Green function of each self-adjoint extension of the operator $L_{0}$ is the Hilbert-Schmidt kernel and is a meromorphic function of $\lambda$. This implies that the spectrum of each self-adjoint extension of the operator $L_{0}$ is discrete.

Assume that $\nu=0$ and let us show that

$$
\overline{\operatorname{det}\left(A_{0}(\lambda)-(-\bar{z}) I_{2 n}\right)}=\operatorname{det}\left(A_{0}(\bar{\lambda})-z I_{2 n}\right) \text {. }
$$

Indeed, the identity

$$
\begin{aligned}
\operatorname{det}\left(A_{0}(\bar{\lambda})-z I_{2 n}\right)= & \operatorname{det}\left(-R_{0}^{*}-(1 / 2+z) I_{n}\right) \\
& \cdot \operatorname{det}\left(R_{0}+(1 / 2-z) I_{n}-P_{0}\left(-R_{0}^{*}-(1 / 2+z) I_{n}\right)^{-1}\left(Q_{0}-\bar{\lambda} I_{n}\right)\right)
\end{aligned}
$$

holds. On the other hand, employing the properties of $P_{0}, Q_{0}, R_{0}$, of the transposed matrices and determinants, it is easy to see that

$$
\overline{\operatorname{det}\left(A_{0}(\lambda)-(-\bar{z}) I_{2 n}\right)}
$$

also satisfies the above formula. Thus, identity (12) holds true. 
Let $\lambda$ be a non-real number and the number of the roots of the equation

$$
\operatorname{det}\left(A_{0}(\bar{\lambda})-z I_{2 n}\right)=0
$$

obeying the condition $\operatorname{Re} z<0$ is equal to $k$ counting the multiplicities. It follows from identity (12) that the number of the roots of the equation

$$
\operatorname{det}\left(A_{0}(\lambda)-(-\bar{z}) I_{2 n}\right)=0
$$

such that $\operatorname{Re} z \leqslant 0$ is equal to $2 n-k$ counting the multiplicities, that is, the deficiency number $n_{+}$and $n_{-}$satisfy the inequality $n_{+}+n_{-} \leqslant 2 n$. Taking into consideration that $n_{+} \geqslant n, n_{-} \geqslant n$, we obtain $n_{+}=n_{-}=n$. The proof is complete.

3.3. Assume that the expression $l[y]$ is defined by the formula

$$
l[y]=-\left(\mathcal{P}_{0} y^{\prime}\right)^{\prime}+i\left(\left(\mathcal{Q}_{0} y\right)^{\prime}+\mathcal{Q}_{0} y^{\prime}\right)+\mathcal{P}_{1}^{\prime} y,
$$

see (5), and assume that the coefficients $\mathcal{P}_{0}(x), \mathcal{P}_{1}(x), \mathcal{Q}_{0}(x)$ of this expression satisfy the following condition.

Condition $\mathbf{B}^{\prime}$. For each $x \geqslant 1$ and some real $\nu \geqslant 0$

$$
\mathcal{P}_{0}^{-1}(x)=x^{-\nu-2}\left(P_{0}^{0}+P_{0}^{1}(x)\right), \quad \mathcal{P}_{1}(x)=x^{\nu+1}\left(P_{1}^{0}+P_{1}^{1}(x)\right), \quad \mathcal{Q}_{0}(x)=x^{\nu+1}\left(Q_{0}^{0}+Q_{0}^{1}(x)\right),
$$

where $P_{0}^{0}, P_{1}^{0}, Q_{0}^{0}$ and $P_{0}^{1}(x), P_{1}^{1}(x), Q_{0}^{1}(x)$ are constant Hermitian matrices and Hermitian matrix functions of order $n$, respectively. Let $\operatorname{det} P_{0}^{0} \neq 0$ and moreover,

$$
\begin{aligned}
& \int_{1}^{+\infty} \frac{\ln ^{r} x}{x}\left(\left\|P_{i}^{1}(x)\right\|+\left\|P_{i}^{1}(x)\right\|^{2}\right) d x<+\infty, \quad i=0,1, \\
& \int_{1}^{+\infty} \frac{\ln ^{r} x}{x}\left(\left\|Q_{0}^{1}(x)\right\|+\left\|Q_{0}^{1}(x)\right\|^{2}\right) d x<+\infty,
\end{aligned}
$$

where $r+1$ is the maximal number of the rows for all Jordan blocks $J_{k}, k \geqslant 1$, in the canonical form of the matrix

$$
A_{\nu}:=\left(\begin{array}{cc}
P_{0}^{0} \phi_{0}+\frac{1}{2} I_{n} & P_{0}^{0} \\
-\phi_{0}^{*} P_{0}^{0} \phi_{0}-\lambda \chi(\nu) I_{n} & -\phi_{0}^{*} P_{0}^{0}-\left(\nu+\frac{1}{2}\right) I_{n}
\end{array}\right),
$$

where $\chi(\nu)=0$ as $\nu>0$ and $\chi(0)=1$, while $\phi_{0}=P_{1}^{0}+i Q_{0}^{0}, \phi_{0}^{*}=P_{1}^{0}-i Q_{0}^{0}$.

It follows from Condition $\left(\mathrm{B}^{\prime}\right)$ that the coefficients $P=\mathcal{P}_{0}, Q=-\varphi^{*} \mathcal{P}_{0}^{-1} \varphi$ and $R=\mathcal{P}_{0}^{-1} \varphi$ in expression (1) in the present case satisfy Condition (B) of Subsection 3.2 and, hence, Theorem 2 remains true. Applying this theorem, we obtain the following theorem.

Theorem 3. Assume that the matrix coefficients $\mathcal{P}_{0}^{-1}, \mathcal{P}_{1}$ and $\mathcal{Q}_{0}$ of the expression l[y] (see (5)) satisfy Condition $\left(B^{\prime}\right)$. Then Statements 1 and 2 of Theorem 2 are true for the equation $l[y]=\lambda y$.

\section{EXAMPLES}

4.1. In the proof of Theorem 2 we have obtained the asymptotic formulae for some fundamental system of solutions of equation (3) as $x \rightarrow \infty$ and these asymptotic formulae are true without assumption that the coefficients of the expression $l[y]$ are Hermitian. They are surely of an independent interest.

Below we give the formulation of the corresponding result for a particular simplest case of expression (5), namely, the following theorem holds true. 
Theorem 4. Let $>0, P_{0}$ be a constant matrix such that $\operatorname{det} P_{0} \neq 0$, the matrix function $P_{1}(x)$ satisfies the condition $x^{-1}\left(\left\|P_{1}(x)\right\|+\left\|P_{1}(x)\right\|^{2}\right) \in L^{1}(I)$ and $\lambda$ is a non-zero complex number. Then the vector equation

$$
\left(x^{\nu+2} P_{0} y^{\prime}\right)^{\prime}+\left(x^{\nu+1} P_{1}(x)\right)^{\prime} y=\lambda y
$$

has a fundamental system of solutions $y_{j}(j=1,2, \ldots, 2 n)$, which as $x \rightarrow \infty$ can be represented as

$$
y_{j}=c_{j}+o(1), \quad y_{n+j}=x^{-(\nu+1)}\left(c_{n+j}+o(1)\right),
$$

where $c_{j}$ and $c_{n+j}(j=1,2, \ldots, n)$ are linearly independent systems of $n$-dimensional vectors.

We point out that Theorem 4 ensures the above given asymptotic formulae for the vector equation $l[y]=\lambda y$, where

$$
l[y](x)=-\left(P y^{\prime}\right)^{\prime}+Q y, \quad x \in I,
$$

$P(x)=x^{\nu+2} P_{0}$ and $Q(x)=\left(x^{\nu+1} P_{1}(x)\right)^{\prime}$. Thus, the coefficient $Q$ in expression (15) can be highly oscillating.

4.2. We provide some particular examples of realization of various deficiency numbers for the operator $L_{0}$. In expression (1) we let $n=2$ and $R(x)=O$. Then it is written as (15) and the matrix functions $P(x)$ and $Q(x)$ satisfy Condition (B) of Subsection 3.2. Simple calculations show that

$$
\mathcal{F}(z, \nu)=\left[\left(z+\frac{\nu}{2}\right)^{2}-\left(\frac{\nu+1}{2}\right)^{2}\right]^{2}-\left[\left(z+\frac{\nu}{2}\right)^{2}-\left(\frac{\nu+1}{2}\right)^{2}\right] \operatorname{sp}\left(P_{0} Q_{0}\right)+\operatorname{det}\left(P_{0} Q_{0}\right) .
$$

The polynomial $\mathcal{F}(z, \nu)$ is obviously an arbitrary quadratic trinomial w.r.t. $\left[\left(z+\frac{\nu}{2}\right)^{2}-\left(\frac{\nu+1}{2}\right)^{2}\right]$ and this is why by choosing the entries of the constant matrices $P_{0}$ and $Q_{0}$, the number of the roots of this polynomial can be any of the numbers 2,3 and 4 . Thus, it is easy to construct examples with minimal, not maximal and maximal deficiency index for the operator $L_{0}$. For instance, letting $\operatorname{sp}\left(P_{0} Q_{0}\right)=0$ and $\operatorname{det} Q_{0}=0$, we obtain the limit-point case for the operator $L_{0}$. Assume now that $\operatorname{sp}\left(P_{0} Q_{0}\right)=-2$ and $\operatorname{det}\left(P_{0} Q_{0}\right)=1$, then the deficiency index of the operator $L_{0}$ is equal to $(4,4)$ as $0<\nu<1$ and to $(3,3)$ as $\nu \geqslant 1$.

\section{BIBLIOGRAPHY}

1. S.A. Orlov. On deficiency index of linear differential operators // Dokl. Akad. Nauk SSSR. 92:3, 483-486 (1953). (in Russian).

2. F.A. Neimark. On the deficiency index of a differential operator // Uspekhi Matem. Nauk. 17:4, 157-163 (1962). (in Russian).

3. R.B. Paris, A.D. Wood. On the $L^{2}(I)$ nature of solutions of $n-$ th order symmetric differential operator and McLeod's conjecture // Proc. Roy. Soc. Edinburg. Sect. A. 90:3-4, 209-236 (1981).

4. R.M. Kauffman On the limit-n classification of ordinary differential operators with positive coefficients // Proc. London Math. Soc. s3-35:3, 496-526 (1977).

5. R.B. Paris, A.D. Wood. Asymptotics of high order differential equations. Pitman Research Notes in Mathematics Series. Longman Scientific \& Technical, Harlow with John Wiley \& Sons, New York. 129. (1986).

6. K.A. Mirzoev. Orlov's theorem on the deficiency index of differential operators // Dokl. Akad. Nauk. 380:5, 591-595 (2001). [Dokl. Math. 64:2, 236-240 (2001).]

7. I.N. Dolgikh, K.A. Mirzoev. Deficiency indices and spectrum of self-adjoint extensions of some classes of differential operators // Matem. Sborn. 127:4, 53-74 (2006). [Sb. Math. 197:4, 525-546 (2006).] 
8. I.N. Braeutigam, K.A. Mirzoev, T.A. Safonova. An Analog of Orlov's theorem on the deficiency index of second-order differential operators // Matem. Zametki. 97:2, 314-317 (2015). [Math. Notes. 97:2, 300-303 (2015).]

9. K.A. Mirzoev, T. A. Safonova. The singular Sturm-Liouville operators with nonsmooth potentials in a space of vector functions // Ufimsk. Matem. Zhurn. 3:3, 105-119 (2011). [Ufa Math. J. 3:3, 102-115 (2011).]

10. W.N. Everitt, L. Marcus. Boundary value problems and sympletic algebra for ordinary differential and quasi-differentrial operators. Math. Surv. Monog. Amer. Math. Soc. Providence, RI. 61 (1999).

11. R.L. Anderson. Limit-point and limit-circle criteria for a class of singular symmetric differential operators // Canad. J. Math. 28:5, 905-914 (1976).

12. M.A. Naimark. Linear differential operators. Nauka, Moscow (1969). [Harrap, London (1968).]

13. A. Zettl. Sturm-Liouville theory. Math. Surv. Monog. Amer. Math. Soc. Providence, RI. 121. (2005).

14. A.M. Savchuk, A.A. Shkalikov. Sturm-Liouville operators with singular potentials // Matem. Zametki. 66:6, 897-912 (1999). [Math. Notes. 66:6, 714-753 (1999).]

15. A.M. Savchuk, A.A. Shkalikov. Sturm-Liouville operators with distribution potentials // Trudy Mosk. Matem. Obsch. 64, 159-212 (2003). [Translat. Mosc. Math. Soc. 64, 143-192 (2003).]

16. S. Faedo. Proprieta asintotiche delle soluzioni dei sistemi differenziali lineari omogenei // Ann. Mat. Pura Appl. Ser. IV. 26, 207-215 (1947).

17. E.A. Coddington, N. Levinson. Theory of ordinary differential equations. McGill-Hill Book Company, New York (1955).

Irina Nikolaevna Braeutigam,

Northern (Arctic) Federal University

named after M.V. Lomonosov,

Severnaya Dvina Emb. 17,

163002, Arkhangelsk, Russia

E-mail: irinadolgih@rambler.ru

Karakhan Agahan ogly Mirzoev,

Lomonosov Moscow State University,

Leninskie Gory, 1,

119991, Moscow, Russia

E-mail: mirzoev.karahan@mail.ru

Tatyana Anatolievna Safonova,

Northern (Arctic) Federal University

named after M.V. Lomonosov,

Severnaya Dvina Emb. 17,

163002, Arkhangelsk, Russia

E-mail: tanya.strelkova@rambler.ru 